\title{
Emploi et précarité des jeunes en Espagne
}

La precariedad del empleo entre los jóvenes: el caso español

Bad Jobs and Young People: the Spanish Case

\section{Luis Enrique Alonso et Carlos Fernández Rodríguez}

\section{OpenEdition}

\section{Journals}

Édition électronique

URL : http://journals.openedition.org/travailemploi/4269

DOI : 10.4000/travailemploi.4269

ISSN : 1775-416X

Éditeur

DARES - Ministère du Travail

Édition imprimée

Date de publication : 15 septembre 2008

Pagination : 71-80

ISSN : 0224-4365

Référence électronique

Luis Enrique Alonso et Carlos Fernández Rodríguez, «Emploi et précarité des jeunes en Espagne », Travail et Emploi [En ligne], 115 | juillet-septembre 2008, mis en ligne le 18 février 2011, consulté le 01 mai 2019. URL : http://journals.openedition.org/travailemploi/4269; DOI : 10.4000/travailemploi.4269 


\title{
Emploi et précarité des jeunes en Espagne
}

\author{
Luis Enrique Alonso, Carlos Fernández Rodríguez (*)
}

L'Espagne a connu en 2006 plusieurs mouvements de manifestation chez les jeunes: la plupart étaient organisées spontanément, par appels téléphoniques, et rassemblèrent beaucoup moins de manifestants qu'en France au mois de mars de la même année. Mais des milliers de jeunes descendirent dans la rue pour protester contre les difficultés d'accès au logement ou contre la précarité de leurs conditions de travail. Depuis, plusieurs autres manifestations de ce type ont suivies, mais avec moins de succès en dépit de l'ampleur du problème. Le prix du logement est aujourd'hui, en Espagne, tout simplement stupéfiant: pour nombre de jeunes espagnols, cela se traduit par un frein à leur indépendance. Cependant, il n'y a pas que les prix du logement qui rendent toute émancipation plus difficile: s'y ajoutent les bas niveaux de salaire, bloqués pour la plupart des moins de trente ans aux environs de 1000 euros par mois (d'où le surnom de «milleuristes»). Si l'on en croit les rapports officiels et les discours politiques, ces nouvelles générations sont pourtant les plus compétentes et les plus qualifiées que le pays ait jamais connues. Malgré cela, l'accès à des emplois de qualité ne leur semble pas ouvert, au sein d'un marché de l'emploi où la précarité ne cesse d'augmenter, touchant plus particulièrement certains groupes sociaux dont les nouvelles générations. Le graphique 1 met en évidence le fait que le pouvoir d'achat des bas salaires n'a pas augmenté au cours des dernières années, ainsi que les fortes différences de salaires entre différentes tranches d'âge.

Il faut noter que les salaires moyens des moins de 26 ans ne représentent qu'un tiers de la rémunération moyenne des plus de 35 ans. Les salaires moyens des 26-35 ans sont très bas comparativement à ceux de la plupart des États membres de l'Union européenne (exception faite des ex-pays du bloc de l'Est), ce qui laisse peu de possibilité d'accéder à l'émancipation et à l'indépendance économique. Les jeunes restent chez leurs parents. Cette situation, toutefois, n'est apparue que dans les années 1980. Au cours

Département de sociologie, université autonome de Madrid; luis.alonso@uam.es, carlos.fernandez@uam.es
Graphique 1 : Salaires annuels par tranches d'âge (en euros constants, année de référence 2000)

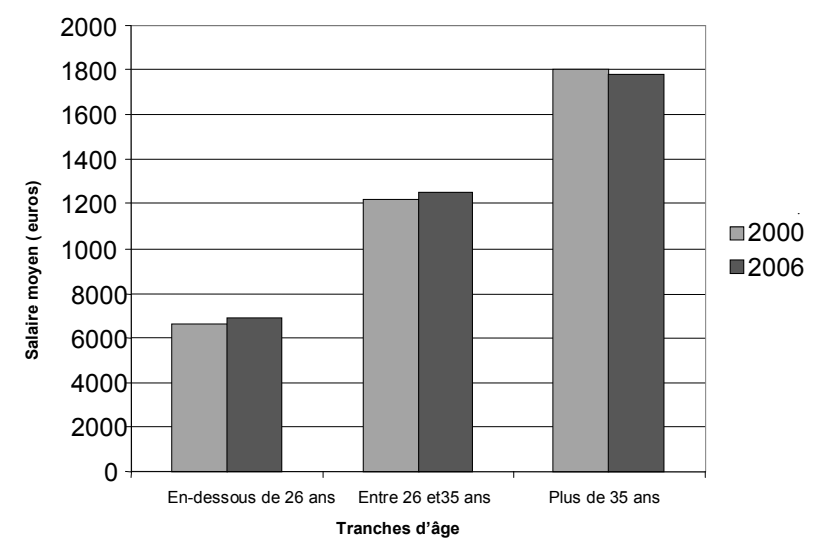

Source: COLECTIVO IOÉ, 2008: 126.

des années 1960-1970, et malgré les faibles salaires de l'époque, les jeunes prenaient leur indépendance beaucoup plus tôt: il est important de mettre ces questions en perspective avec les transformations que l'économie et la société espagnole ont connues dans les dernières décennies.

Le passage du fordisme au postfordisme s'est traduit, pour les économies occidentales, par le renforcement et le développement du secteur tertiaire et par la restructuration drastique du secteur industriel. Le déclin de nombreux secteurs industriels traditionnels a incité les pouvoirs publics à prendre des mesures moins radicales pour éviter le chômage de masse, comme le recours à des contrats de travail plus flexibles ou à des licenciements partiels de la main-d'œuvre (KoCH, 2006). Au cours des trois dernières décennies, ces politiques ont été largement mises en œuvre par les entreprises pour améliorer leur compétitivité. En ce sens, l'Espagne est un cas paradigmatique, la précarité y ayant lourdement touché une génération entière de jeunes entrés sur le marché du travail dans les années 1980 (Alonso, Martínez Lucio, 2006). 


\section{Encadré 1 \\ D'un fordisme inachevé au postfordisme}

Le modèle économique espagnol de la fin du XXe siècle a impliqué une rupture avec le modèle d'industrialisation qui prédominait dans les années 1960 et 1970. Ce modèle industriel était tout à la fois dépendant, protectionniste et le fruit d'une politique étatique; il a produit une industrie nationale significative, même si elle était relativement faible sur le plan international, forgée grâce aux excédents technologiques et financiers des marchés internationaux. Les conséquences sociales en Espagne de ce type de développement sont connues: transition d'un pays basé sur l'agriculture à une économie plus développée, urbanisation, ascension de nouvelles classes moyennes dont les revenus provenaient des salaires. Ce processus de développement se fit tardivement, de manière rapide et non-uniforme. L'absence de tous droits fondamentaux, droits sociaux comme droits du travail (refusés par la dictature de Franco), liée aux spécificités du fordisme espagnol (comme système de réglementation et d'ajustements entre une production industrielle de masse et une consommation de masse), en firent un fordisme inachevé ou incomplet. Notre thèse est qu'il fut incomplet parce qu'incapable de générer un modèle de croissance économique efficace et autonome, tout en multipliant les obstacles sur le chemin de la normalisation des conflits liés à la répartition des richesses (processus réprimé par l'autoritarisme du régime franquiste) $)^{(1)}$.

Le fordisme inachevé de l'Espagne a évolué en un fordisme d'un genre particulier au sein duquel le marché du travail s'est segmenté de manière marquée. La restructuration du capitalisme espagnol dans les années 1980 et 1990 a impliqué une profonde désindustrialisation, qui a mis un terme au modèle nationaliste de développement économique. L'État a été le principal acteur de ce processus de remercantilisation de la société, démantelant les secteurs les moins concurrentiels de l'industrie et promouvant l'internationalisation de l'économie espagnole. Les centres de décision économique se trouvent maintenant à l'étranger et l'économie espagnole est devenue une économie de services (CuADRADO, 1990). Ceci a créé un cadre où des vagues de restructurations industrielles se sont succédées, amenant dans leur sillage des adaptations rigoureuses et souples à la fois, et une restructuration financière portée par l'État. La réforme profonde du droit du contrat de travail (le travail est désormais temporaire ${ }^{(2)}$, sans garantie de le conserver, et beaucoup plus diversifié et fragmenté) et l'arrivée massive des capitaux internationaux ont créé un type d'économie très différent de celui des années 1960-1970. L'économie espagnole a connu un repositionnement dans la division et la répartition internationale du travail. Elle est passée d'une économie semi-périphérique accusant un retard, à une économie semi-périphérique avancée au sein du système international (ALONSO, CondE, 1997). II est vrai que certaines entreprises (Inditex, Telefónica, Santander) ont acquis un rang international et que le pays est considéré comme la huitième économie mondiale (en concurrence avec le Canada). Mais, en dépit de visions triomphalistes qui ont tenté d'offrir l'image d'un nouveau «miracle économique espagnol », la réalité est plus diffuse, inégale et fragile. Ceci s'explique par l'influence de facteurs tels que les inégalités et le retard économique historique de l'Espagne - avec de fortes différences régionales, des zones historiquement dépendantes présentant des surfaces agraires et urbaines sous-développées et des coûts sociaux liés à ces processus de restructuration industrielle - le marché noir, les nouvelles situations d'indigence, le taux élevé du chômage structurel, la dépression économique enregistrée par d'anciens secteurs industriels, etc. (3).

\footnotetext{
(1) Voir Muñoz, Roldán, SERRANO (1978) pour une première analyse de ce processus. La définition du fordisme espagnol comme «fordisme inachevé» figure dans l'ouvrage de ToHARIA (1986), devenu un classique. D'autres auteurs ont qualifié le cas espagnol de «fordisme attardé» (Koch, 2006).

(2) La terminologie espagnole regroupe sous le terme de temporaire la totalité des situations contractuelles de courte durée, intérimaire ou autres, et caractérisées par la précarité.

(3) Une vision très incisive et perspicace de cette première et longue vague du processus a été décrite par Etxezar reta (1991).
}

La nouvelle structure économique de l'Espagne a eu pour conséquence d'engendrer un marché de l'emploi spécifique. Les pertes d'emploi dans le secteur industriel et la croissance du secteur des services ont créé une très forte concentration d'effectifs dans le secteur tertiaire. Le marché du travail a subi une segmentation très large. D'une part, les emplois du tertiaire - comme l'a démontré empiriquement MuÑoz DE Bustillo (1993) - sont temporaires (1) et externalisés, avec des contrats non stables et de faible qualité. Il existe d'autre part un noyau dur de postes nécessitant de hautes qualifications - principalement dans les domaines de l'innovation et de

(1) Voir encadré 1 note 2. la gestion financière - qui tentent de répondre aux exigences de concurrence et de rentabilité maximales imposées par le nouveau système postindustriel. Quoi qu'il en soit, cette précarité du marché de l'emploi n'a pas mis fin aux habitudes de consommation des plus jeunes générations. Cet article examine de plus près les emplois de faible qualité et la précarité qui semblent caractériser le marché espagnol du travail. Nous nous attacherons, dans un premier temps, à dégager les grands traits des réformes du marché du travail adoptées depuis le passage à la démocratie (pour plus d'approfondissement, voir Miguélez et Prieto supra) et qui forment le cadre de l'évolution de l'emploi des jeunes. Puis nous décrirons la situation que connaissent les jeunes et réfléchirons à l'impact de la précarité du marché du travail sur la vie des 
jeunes en Espagne. Nous nous donnons également pour objectif de mettre en lumière les principales caractéristiques de la transition espagnole vers un modèle postfordiste, dans lequel les inégalités du marché du travail semblent avoir un effet sur l'accès au logement, mais ne semblent pas avoir mis de frein aux habitudes de consommation comme valeur idéologique essentielle.

\section{Les réformes du marché du travail: des emplois précaires pour les jeunes}

Le phénomène de la précarité sur le marché du travail espagnol a été largement étudié (MiguÉLEZ, Prieto, 1999; Alonso, 1999 et 2001; López Calle, Castillo, 2004), du fait de l'extraordinaire impact qu'elle a eu sur la société espagnole. Depuis le début des années 1980, l'âge de l'émancipation et de l'accès à l'indépendance économique n'a cessé de reculer, le taux de la natalité ayant, pour sa part, énormément régressé, et les longues durées de travail quotidien, dans le secteur privé de l'économie, étant acceptées comme pratique courante et usuelle. Simultanément, la précarité s'est répandue à un point tel que l'Espagne est devenue le pays de l'Union européenne affichant le taux le plus élevé en termes de travail temporaire. Alors que la génération correspondant au stade du fordisme inachevé était extrêmement protégée par la législation paternaliste dite Fuero del Trabajo (Code du travail sous le régime franquiste, ratifié en 1938 afin de créer un état corporatiste), les carrières professionnelles des jeunes générations n'ont rien gagné en matière de stabilité. Selon des publications consacrées depuis le début des années 1980 à l'analyse de la relation entre logement et précarité du marché du travail (par exemple, LEAL, 1987, 2003 et 2004; LeAl, CoRTÉs, 1995; CoRTÉs, 2005; voir aussi de nombreux articles parus dans la revue Ciudad y Territorio et des rapports institutionnels mais souvent critiques sur de nombreux points, parus dans le magazine Instituto de la Juventud), le marché espagnol du travail et ses difficultés semblent avoir une forte influence sur la difficile émancipation des jeunes, étant donné leur incapacité à assumer un loyer ou un prêt immobilier. Nous donnerons un aperçu général de l'évolution du marché du travail espagnol pour les jeunes générations et expliquerons comment la précarité est devenue partie intégrante du paysage social.

En 1977, deux ans après le décès de Franco et quelques mois après les premières élections démocratiques depuis 1936, des accords politiques furent finalement trouvés (les accords de Moncloa dit pacte de la Moncloa) pour tenter de faire face à la difficile situation économique de l'époque: la dette extérieure était colossale, le taux d'inflation atteignait les $44 \%$ en 1977 et l'endettement des entreprises

\section{Encadré 2 \\ L'Espagne et la démocratie}

L’Espagne a hérité du régime franquiste un marché du travail extrêmement rigide, afin de garantir l'adhésion de la main-d'œuvre au nouvel État corporatiste d'inspiration fasciste qui a vu le jour à la suite de la victoire des nationalistes lors la guerre civile espagnole. L'expansion économique des années 1960 s'accompagna d'une situation de plein-emploi (partiellement obtenue par l'émigration d'un grand nombre de travailleurs espagnols à destination de l'Europe centrale et du Nord, pour y occuper des emplois demandant très peu de qualifications). La croissance économique, l'industrialisation et le développement d'une économie de services tirée par le tourisme contribuèrent à l'émergence d'une nouvelle classe moyenne, modifiant considérablement la société et ses valeurs. L'Espagne catholique, traditionnelle et agraire se transforma peu à peu en une société moderne de consommation de masse, en dépit de certains manques liés à un contexte politique où régnait l'autoritarisme. Le niveau des revenus s'améliora et le secteur du bâtiment fut alimenté par des demandes constantes en logements. À la fin des années 1960, le pays connut même un baby-boom, favorisé par la prise d'indépendance, plus précoce, d'une génération parvenant sur un marché du travail où il n'existait quasiment aucun chômage. Le marché du travail, sous Franco, apportait la stabilité de l'emploi, dans un contexte paternaliste et une économie où peu de femmes travaillaient en dehors du foyer.

Cependant, la crise pétrolière vint considérablement modifier ces perspectives, comme pour le reste du monde occidental. L'Espagne, du fait de sa dépendance énergétique, fut fortement touchée par le choc pétrolier qui se produisit à un moment où de considérables changements politiques s'opéraient. Les dernières années du régime franquiste eurent à faire face à une opposition politique croissante (révoltes étudiantes, syndicats illégaux) en même temps qu'à la crise économique, mal gérée. Les mauvais résultats des secteurs industriels, comparés à ceux des nouveaux concurrents internationaux, générèrent faillites et fermetures d'usines et le chômage et l'inflation se mirent à augmenter. Le chômage des jeunes augmentait simultanément dans le reste de l'Europe (devenant un problème critique: voir par exemple CASSON, 1979; OCDE, 1984), l'Espagne n'y faisant pas exception. Qui plus est, cette période d'instabilité politique empêcha les premiers gouvernements post-franquistes de prendre des décisions économiques difficiles.

était excessivement élevé. Le second point du pacte de la Moncloa comportait des mesures en faveur de l'emploi des jeunes et promulguait un nouveau type de relation contractuelle, considérée comme «expérimentale», qui avait pour but de «promouvoir 
l'emploi des jeunes » grâce à des contrats de deux ans dont les cotisations de Sécurité sociale étaient partiellement financées (à hauteur de 50\%) par les pouvoirs publics (Présidence du Gouvernement, 1977). Ces contrats temporaires étaient considérés comme devant être «exceptionnels» et ne ciblaient que les individus postulant à un premier emploi. Toutefois, sur le marché du travail espagnol, ces mesures peuvent être considérées comme à l'origine de la précarisation: de fait, toutes les réformes du marché de l'emploi jusqu'en 1997 ne firent qu'aggraver les conditions de travail des jeunes générations. Ces nouvelles politiques n'aidèrent pas à résoudre le problème du chômage. À l'inverse, si le taux du chômage, à l'époque du pacte de la Moncloa, était de 5,69\%, il dépassait 10\% au début des années 1980. Progressivement, les contrats temporaires devinrent l'arme utilisée contre le chômage massif qui affectait les jeunes plus que tout autre groupe social. En 1980, le Statut des salariés (nouveau cadre réglementant les droits des salariés) laissa également le champ libre à de nouveaux modes de relations contractuelles, qui furent pleinement développés ultérieurement(2) (Bilbao, 1989; Alonso, 2007).

Au cours des années 1980, le taux de chômage ne cessa d'augmenter: en 1984, il dépassait les $20 \%$, marquant l'une des pires périodes qu'ait eu à connaître le marché du travail espagnol (LAPARRA, 2006). Le nouveau gouvernement social-démocrate du Parti socialiste ouvrier espagnol (PSOE) avait remporté avec une confortable avance les élections de 1982 et ses dirigeants (dont Felipe González, président du parti) adoptèrent une approche politicoéconomique plutôt qu'une approche capitalistique (КocH, 2006). En 1984, le gouvernement se lança dans une importante réforme, dite Ley de Fomento del Empleo (loi sur le développement et la promotion de l'emploi), qui retenait quatorze types de contrats, pour la plupart à durée déterminée. Certains visaient spécifiquement les jeunes: les plus importants étaient le contrat de formation et le contrat de stage. Ce fut le point de départ d'un marché du travail à deux vitesses: les salariés en contrat à durée indéterminée n'étaient pas touchés par cette réforme; ceux qui avaient des contrats temporaires de travail supportèrent les coûts sociaux de la réforme, les entreprises préférant les garder dans leurs effectifs en raison de coûts de licenciement moindres. Ceci eut un impact tragique sur l'existence de nombreux salariés et, en particulier, sur celle des générations les plus jeunes

(2) Le Statut des salariés, dans sa première version, n'évoquait que le contrat de travail temporaire et l'assortissait de trois conditions : il ne pouvait concerner que certaines catégories de salariés; une limite était fixée au nombre des contrats temporaires qu'une même entreprise pouvait signer, en fonction du nombre d'emplois stables qu'elle comportait; enfin, seuls les contrats nouvellement signés étaient concernés, ce qui protégeait les CDD précédemment conclus. Ce dernier point signifiait que les jeunes seraient gravement touchés par ces futurs contrats temporaires, comme nous l'expliquerons plus loin.
(LóPez CAlle, CAstillo, 2004), et contribua au développement du nouveau concept de «jeunesse de longue durée» (SANTOS, 2003). Ces contrats remportèrent un vif succès auprès des entreprises, contribuant à accroître la précarité parmi les personnes les plus vulnérables du marché de l'emploi: les jeunes, les femmes et les chômeurs. De 1987 à 1995, le pourcentage des moins de 30 ans titulaires d'un contrat à durée à durée déterminée passa de $27,4 \%$ à $63,1 \%$ (Consejo de la Juventud, 2007).

L'Espagne rejoignit la Communauté économique européenne en 1986; elle connut alors une période d'essor économique pendant laquelle son taux de chômage décrut légèrement à partir de 1987 (sans jamais toutefois pouvoir passer sous la barre des 15\%). En 1988, les politiques d'assouplissement furent renforcées par une nouvelle loi, dite Programme d'emploi pour les jeunes. Les syndicats attaquèrent de front cette nouvelle législation, dont la principale mesure était un nouveau contrat d'insertion pour les jeunes, connu sous le nom de Contrato Basura (contrat poubelle). L'épisode se conclut par une grève générale, couronnée de succès, en 1988, et par un désaccord croissant entre le gouvernement et les syndicats. Ceci fut d'abord considéré comme une victoire des syndicats du fait de la non-application de la nouvelle législation (LAPARRA, 2006). Mais une réforme plus profonde devait voir le jour quelques années plus tard, à la suite d'une soudaine récession qui détruisit de nombreux emplois en 1994, portant le taux de chômage au niveau record de $24,5 \%$. La loi de novembre 1994 introduisit plusieurs réformes destinées à donner davantage de flexibilité au marché du travail (BAYLOS, 1996). Ces réformes visaient à nouveau les jeunes générations: les anciens contrats de formation étaient remplacés par des contrats d'apprentissage. Plus important, les relations de travail voyaient l'émergence d'un nouvel acteur, les entreprises de travail intérimaire, qui seront essentielles pour développer l'externalisation des relations de travail dans l'économie espagnole, et l'apparition de nouveaux contrats intérimaires de travail. Â nouveau, les jeunes furent les plus touchés, et devinrent la principale main-d'œuvre de ces entreprises. En dépit des nouvelles mesures, le chômage ne baissa qu'assez faiblement et la précarité du travail atteignit des limites historiques: en 1995, le taux de chômage était encore supérieur à $20 \%$ et plus de $35 \%$ des contrats relevaient du travail temporaire (avec plus de la moitié détenue par des jeunes: 63,1\% d'entre eux étaient alors titulaires d'un contrat à durée déterminée).

En 1996, le PSOE perdit les élections et le Parti populaire, parti conservateur, forma un nouveau gouvernement sous la présidence de José María Aznar. Des accords furent négociés avec les syndicats et les associations d'employeurs, se soldant en 1997 par une nouvelle réforme du travail (ALBIOL et al., 1998). Cette réforme comprenait plusieurs accords, dont l'un prévoyait des avantages pour les entreprises embauchant de nouveaux salariés 
sur des contrats à durée indéterminée, et des coûts inférieurs en cas de licenciement. L'ancien contrat temporaire visant à favoriser l'emploi (CDD) fut également supprimé. Les contrats d'apprentissage furent remplacés par de nouveaux contrats de formation, où la formation avait une part réellement substantielle. Ainsi, les conditions de travail des jeunes semblèrent s'améliorer légèrement, par l'introduction d'une plus grande flexibilité en même temps que par la promotion d'une plus grande stabilité, conformément à la doctrine de flexicurité et de partenariat prônée par l'Union européenne. Cette réforme portait atteinte à une longue tradition où travail temporaire et précarité se côtoyaient, tout en représentant une nouvelle avancée vers un marché du travail libéré. Elle fut considérée comme un succès relatif, le taux de chômage commençant à baisser, alors même que parallèlement le contexte indiquait clairement un nouvel élan économique (3) (LAPARRA, 2006). En fait, en 2004, les contrats temporaires de travail, concernant les moins de 30 ans, ne représentaient «plus que $52,5 \%$ d'entre eux.

Le PSOE revint au pouvoir en 2004 et le nouveau cabinet conduit par José Luis Rodríguez Zapatero entreprit une réforme du marché du travail encore en vigueur, après de nouvelles négociations avec les syndicats et les associations d'employeurs. La nouvelle réforme de 2006 tentait à nouveau d'améliorer la stabilité de l'emploi en réduisant les coûts liés au licenciement. Un effort important fut fait pour éradiquer les abus liés aux contrats temporaires et assurer un meilleur contrôle de la situation, bien que deux types de contrats temporaires soient maintenus : le contrat de mission et le contrat en fonction des besoins de la production. Quoi qu'il en soit, les perspectives d'avenir restent sombres, car la croissance économique au cours des prochaines années semble devoir être plus faible, non seulement du fait du ralentissement enregistré par le secteur de la construction et du bâtiment (essentiel en Espagne), mais également du fait des turbulences financières mondiales. D'après les dernières données disponibles (2006), les contrats temporaires concernent encore près de 55,2\% des moins de 30 ans (CONSEJO DE LA JUVENTUD, 2007).

(3) En 2001 furent introduites de nouvelles réformes étendant les conventions collectives susceptibles de s'appliquer à ces nouveaux contrats à durée indéterminée et une législation plus spécialisée et plus complexe, applicable aux contrats à temps partiel, vit le jour. En 2002, toutefois, eut lieu une nouvelle tentative visant à apporter des changements à la législation du travail en dehors du cadre de la concertation sociale cette fois, avec des dispositions autoritaires également plus marquées. Ces mesures visaient à rendre moins onéreux les coûts d'un licenciement, ainsi qu'à réduire les droits des personnes sans emploi. L'un des points qui fit le plus polémique était l'obligation pour les chômeurs d'accepter n'importe quel type d'emploi, sous peine de se voir supprimer leurs allocations chômage. Ceci aboutit en 2002 à une autre grève générale, qui remporta un succès modéré. Mais suite à cette grève, le gouvernement reconsidéra sa position et abandonna cette proposition.

\section{Encadré 3 \\ Les réformes du marché du travail en Espagne}

Le volet principal de ces réformes fut la mise en place du contrat temporaire de travail - auquel les entreprises ont largement recouru (au point d'en faire un usage abusif) - et des contrats d'apprentissage, contrats de formation et contrats de stage, toutes mesures présentées comme devant lutter contre le chômage des jeunes. Depuis, une désorganisation du secteur de la production s'est produite, avec la fermeture de la plupart des entreprises du secteur industriel public et le passage à une société orientée vers les services. De nombreux droits sociaux du travail ont été érodés par les nouvelles politiques, qui tendaient exclusivement à rendre le marché du travail plus flexible (UGT, 2004; SAntos, 2005). Au cours de la dernière décennie, l'accès au marché du travail s'est amélioré, parallèlement toutefois à une dégradation des conditions de travail (ColECTIVo IOÉ, 2008: 133). Les jeunes ont été fortement touchés par ces réformes, qui les ont mis en situation de grande précarité. En dépit des dernières réformes opérées, qui introduisaient des mesures d'incitation au développement de contrats à durée indéterminée, la réalité est que la précarité liée aux contrats temporaires de travail, affiche toujours un taux supérieur à $30 \%$, et même de plus de $50 \%$ parmi les jeunes de moins de 30 ans. Instabilité et flexibilité se sont accompagnées d'une "course effrénée" à l'éducation et à l'enseignement, par laquelle les jeunes générations ont cherché à acquérir certaines compétences (nouvelles technologies, langues étrangères) ou à acquérir des diplômes universitaires.

\section{Jeunes et précarité dans l'Espagne d'aujourd'hui}

Nous nous efforcerons ici de donner une définition de la précarité sur le marché du travail espagnol à partir d'éléments chiffrés, et soulignerons l'existence d'un lien possible entre cette précarité et l'émancipation tardive des jeunes et les problèmes sociaux qu'ils rencontrent.

\section{Données sur le marché du travail espagnol}

L'Espagne est le pays de l'Union européenne qui a le taux le plus élevé de travailleurs temporaires (ou intérimaires) $-34,4 \%$ en 2006 . En 2007, ce taux est le double de celui de l'Union européenne à vingt-sept $(31,8 \%$ contre $14,5 \%)$. Les jeunes sont particulièrement touchés par cette situation (voir tableau 1): 
Tableau 1: Taux d'emploi temporaire des jeunes actifs occupés en 2007

\begin{tabular}{|l|c|}
\hline \multicolumn{1}{|c|}{$\begin{array}{c}\text { Jeunes percevant un salaire } \\
\text { (de 16 à 29 ans) }\end{array}$} & $\begin{array}{c}\mathbf{2 0 0 7} \\
\text { (deuxième trimestre) }\end{array}$ \\
\hline Total & 4470880 \\
\hline $\begin{array}{l}\text { Titulaires d'un contrat à durée } \\
\text { indéterminée }\end{array}$ & 2161588 \\
\hline $\begin{array}{l}\text { \% des jeunes gens titulaires d'un } \\
\text { contrat } \\
\text { à durée indéterminée }\end{array}$ & $48,3 \%$ \\
\hline Titulaires d'un contrat temporaire & 2309292 \\
\hline $\begin{array}{l}\text { \% des jeunes gens titulaires d'un } \\
\text { contrat temporaire }\end{array}$ & $51,7 \%$ \\
\hline
\end{tabular}

Source: Institut national de la statistique espagnol (INE), 2007.

Le nombre de jeunes de moins de 30 ans en situation de travail temporaire atteint le taux de $51,7 \%$ en 2007 (OBSERVATORIO JoVEN DEL EMPLEO EN EsPaÑa, 2007). Le tableau 2 indique les taux d'emploi précaire dans différents secteurs d'activité économique. L'emploi à caractère temporaire, pour ces jeunes et pour chacun de ces secteurs, est deux à trois fois plus important que pour l'ensemble de la population, exception faite du secteur du bâtiment. Le secteur des services est celui qui offre le plus d'emplois aux jeunes générations, mais c'est en même temps un secteur fortement touché par la précarité et le caractère temporaire des postes proposés (presque la moitié des jeunes ont un contrat temporaire de travail dans un secteur qui représente approximativement $70 \%$ de l'emploi global). Dans le secteur de l'administration publique, près de $62,1 \%$ des moins de 30 ans occupent des emplois non stables.

Tableau 2: Taux d'emploi temporaire chez les jeunes actifs par secteur d'activité

\begin{tabular}{|l|c|c|}
\hline \multicolumn{1}{|c|}{ Secteur } & $\begin{array}{c}\text { \% de l'emploi } \\
\text { temporaire par } \\
\text { secteur }\end{array}$ & $\begin{array}{c}\text { \% de l'emploi tempo- } \\
\text { raire par secteur pour } \\
\text { les 16-29 ans }\end{array}$ \\
\hline Industrie & $22,7 \%$ & $45,5 \%$ \\
\hline Bâtiment & $55,6 \%$ & $66,0 \%$ \\
\hline Services & $29,4 \%$ & $48,7 \%$ \\
\hline Agriculture & $59,7 \%$ & $73,3 \%$ \\
\hline $\begin{array}{l}\text { Tous secteurs } \\
\text { confondus }\end{array}$ & $30,7 \%$ & $51,7 \%$ \\
\hline
\end{tabular}

Source: Institut national de la statistique espagnol (INE), 2007).

Il est également important de noter que la distribution géographique de l'emploi précaire à travers l'ensemble du pays n'est pas uniforme. Ainsi, les régions affichant les résultats économiques les plus élevés sont celles où les taux du travail temporaire sont les plus faibles, comme Madrid, la Catalogne ou le Pays Basque. Ceci ne signifie pas pour autant que ces taux soient effectivement bas: la région affichant le taux le plus bas est la Catalogne, avec $26 \%$. Cependant, dans les régions les plus pauvres, comme l'Andalousie, les chiffres atteignent le taux impressionnant de $46 \%$ (COLECTIVo IOÉ, 2008). La répartition de la précarité du travail et son ampleur sont fonction des résultats économiques et de la richesse des régions. En comparaison avec d'autres pays de l'Union européenne (tableau 3), l'Espagne vient en tête, avec un tiers de sa masse salariale touché par la précarité. Le postfordisme modifie profondément les conditions de travail en Europe, où le travail temporaire atteint dans de nombreux pays un taux très élevé pour les jeunes actifs. Dans une société postfordiste, marquée par l'emploi précaire (Alonso, 2001), le travail perd la place centrale qu'il occupait au cœur de la vie sociale, le consumérisme prenant le pas sur lui : sur ce point, l'Espagne est un bon exemple et occupe une place de leader.

Tableau 3 : Taux d'emploi temporaire dans différents pays de l'Union européenne

\begin{tabular}{|l|c|c|}
\hline & $\begin{array}{c}\text { Taux d'emploi } \\
\text { temporaire }\end{array}$ & $\begin{array}{c}\text { Taux d'emploi } \\
\text { temporaire chez les } \\
\text { moins de 25 ans }\end{array}$ \\
\hline Allemagne & $14,2 \%$ & $56,4 \%$ \\
\hline Espagne & $31,8 \%$ & $62,5 \%$ \\
\hline Finlande & $17,3 \%$ & $47,6 \%$ \\
\hline France & $14,3 \%$ & $53,3 \%$ \\
\hline Grèce & $11,1 \%$ & $27 \%$ \\
\hline Irlande & $9 \%$ & $21,4 \%$ \\
\hline Portugal & $22,2 \%$ & $51,6 \%$ \\
\hline $\begin{array}{l}\text { Union européenne } \\
\text { à vingt-cinq }\end{array}$ & $14,5 \%$ & $41,2 \%$ \\
\hline
\end{tabular}

Source: Institut national de la statistique espagnol (INE), 2007)

Ces jeunes générations, rappelons-le, sont celles qui ont suivi l'enseignement scolaire ou universitaire le plus poussé. En raison du chômage massif qui régnait au début des années 1980, la politique de l'enseignement a encouragé la poursuite des études et l'obtention de diplômes, afin que les jeunes bénéficient d'opportunités plus nombreuses sur le marché de l'emploi, auquel ils accèdent en même temps plus tardivement. Le nombre de jeunes possédant un diplôme universitaire atteint un niveau élevé, particulièrement parmi les jeunes filles (voir tableau 4). Si ces chiffres sont proches de ceux d'autres pays européens (à l'exception de l'Allemagne), le contraste avec les générations précédentes est plus important en Espagne. Ceci n'a pas eu pour effet d'empêcher la précarité, mais l'a plutôt accentuée, du fait que les salariés nouvellement diplômés et qualifiés se sont soudain retrouvés sur un marché de l'emploi déjà resserré. Les jeunes titulaires d'un diplôme cherchent alors à ajouter de nouvelles compétences à leur $\mathrm{CV}$, entamant une nouvelle «course effrénée » qui fait que leur diplôme perd de sa valeur sur le marché du travail. 
Tableau 4: Pourcentage des individus titulaires d'un diplôme universitaire en $\mathbf{2 0 0 3}$ par sexe et par tranches d'âge

\begin{tabular}{|l|c|c|c|c|}
\hline \multirow{2}{*}{} & \multicolumn{2}{|c|}{ De 24 à 64 ans } & \multicolumn{2}{c|}{ De 24 à 34 ans } \\
\cline { 2 - 5 } & Hommes & Femmes & Hommes & Femmes \\
\hline Allemagne & $17 \%$ & $12 \%$ & $15 \%$ & $13 \%$ \\
\hline Espagne & $19 \%$ & $17 \%$ & $22 \%$ & $30 \%$ \\
\hline Finlande & $17 \%$ & $16 \%$ & $20 \%$ & $26 \%$ \\
\hline France & $14 \%$ & $14 \%$ & $20 \%$ & $24 \%$ \\
\hline Grèce & $14 \%$ & $12 \%$ & $14 \%$ & $19 \%$ \\
\hline Irlande & $17 \%$ & $16 \%$ & $22 \%$ & $24 \%$ \\
\hline Portugal & $7 \%$ & $10 \%$ & $9 \%$ & $17 \%$ \\
\hline Royaume-Uni & $20 \%$ & $18 \%$ & $25 \%$ & $23 \%$ \\
\hline
\end{tabular}

Source: ministère de l'Éducation, Espagne.

Ces pourcentages élevés font miroir avec le nombre également important d'individus n'ayant accès qu'à un enseignement de premier niveau. Le niveau scolaire des générations précédentes est particulièrement bas en Espagne (à l'avant-dernière place en 2003, avant le Portugal, comme le montre le tableau comparatif 5). Il se fait jour ainsi une situation duelle en termes de formation et d'éducation, avec un pourcentage élevé d'individus n'ayant qu'un faible bagage et un pourcentage relativement élevé aussi de personnes titulaires de diplômes universitaires.

Tableau 5 : Pourcentage d'individus dont le niveau d'enseignement est inférieur à l'enseignement secondaire en 2004

\begin{tabular}{|l|c|c|}
\hline & De 25 à 64 ans & De 25 à 34 ans \\
\hline Allemagne & $16 \%$ & $15 \%$ \\
\hline Espagne & $55 \%$ & $39 \%$ \\
\hline Finlande & $22 \%$ & $11 \%$ \\
\hline France & $35 \%$ & $20 \%$ \\
\hline Grèce & $44 \%$ & $27 \%$ \\
\hline Irlande & $37 \%$ & $21 \%$ \\
\hline Portugal & $75 \%$ & $60 \%$ \\
\hline Royaume-Uni & $35 \%$ & $30 \%$ \\
\hline
\end{tabular}

Source: Institut national de la statistique espagnol (INE), 2006.

Paradoxalement, cette situation n'a pas conduit à un système à deux vitesses. Excepté le cas de certaines personnes ayant accès à un poste très qualifié en raison d 'études supérieures, ou encore en raison de compétences spécifiques ou par relations personnelles, la précarité touche même les titulaires d'un doctorat, ce phénomène «rampant» concernant toutes les catégories des nouvelles générations de salariés. Un nouveau groupe social s'est développé : les «milleuristes », c'est-à-dire les personnes gagnant environ mille euros par mois (EsPIDO FREIRE, 2006). Ces personnes ont des compétences plus poussées que les générations précédentes, mais l'accès à des emplois stables leur est plus difficile, alors qu'elles vivent souvent seules.

\section{Emplois précaires en Espagne: une émancipation tardive}

L'un des traits les plus intéressants du concept de «jeune» tient à son évolution. En Espagne, de nombreux rapports (INJUve, 2005; PÉREZ et al., 2006) concentrent leur analyse sur l'extension de cette tranche d'âge, à laquelle les plus de trente ans sont encore assimilés. De plus en plus de trentenaires dépendent en effet de leurs parents, tant sur un plan économique que sur celui du logement. Pour un nombre toujours plus grand d'entre eux, il ne s'avère pas possible de donner corps à leurs propres projets (autonomes) de vie. Certains discours ont mis en relief l'indolence des jeunes générations, accusant celles-ci de vivre trop à leur aise au sein du cocon familial. Il est vrai que les relations interpersonnelles, au sein de la famille, se sont modifiées: la vie, pour l'enfant (qui devient comme un «produit de luxe» dans cette société de consommation), est de bien des manières plus aisée qu'elle ne l'était pour les générations précédentes, non seulement en termes de richesse économique, mais aussi d'affection. Quoi qu'il en soit, les deux principaux problèmes qui, selon nous, font que les jeunes générations restent chez leurs parents, dans l'incapacité de concevoir des projets autonomes, sont d'abord économiques. Notre hypothèse est que cette situation repose essentiellement sur deux facteurs: les prix élevés du logement et la précarité des emplois (avec les bas salaires que cela entraîne). Les rapports cités plus haut (PéRez et al., 2006: 8) montrent que les jeunes gens aspirent à l'indépendance : $71,4 \%$ des moins de 30 ans habitent encore chez leurs parents (tableau 6), mais $80 \%$ d'entre eux (INJUVE, 2005) aimeraient être indépendants.

Les résultats montrent que la plupart éprouvent apparemment des difficultés à quitter le foyer parental. Il existe manifestement un pourcentage de plus en plus important d'individus parvenant, entre vingt et trente ans, à s'émanciper et à prendre leur indépendance; mais il y a, parallèlement, plus de $20 \%$ des jeunes de 30 à 34 ans qui vivent encore chez leurs parents. L'âge où ces personnes parviennent à prendre leur indépendance s'est même accru, en passant à 36 ans (PÉREZ et al., 2006: 11). Pourquoi les jeunes espagnols quittent-ils si tardivement le foyer parental? Selon certains sociologues, tel que LeAL (2002), les relations familiales et le passage à l'âge adulte, en Europe du Sud (Espagne, Italie, Grèce), sont très différents de ceux des autres pays européens. En dehors du mariage, il existe peu d'options pour quitter le foyer parental, tandis que dans certains pays, tels que le Royaume-Uni, l'Allemagne ou la Finlande, quitter le foyer est un aspect essentiel du passage à la vie adulte. Dans le cas de l'Espagne, les jeunes gens restent chez leurs parents et économisent en vue de l'achat futur de leur premier logement, poursuivant généralement des études universitaires. 
Tableau 6 : Réponses à la question: «quel est votre lieu habituel de résidence?»

\begin{tabular}{|l|r|r|r|r|r|r|r|}
\hline \multirow{2}{*}{} & \multirow{2}{*}{ Total } & \multicolumn{2}{|c|}{ Sexe } & \multicolumn{3}{|c|}{ Tranches d'âge (4) } \\
\cline { 3 - 8 } & & Hommes & Femmes & $18-19$ & $20-24$ & $25-29$ & $30-34$ \\
\hline $\mathbf{N}=$ & $\mathbf{( 1 4 6 6 )}$ & $\mathbf{( 7 4 5 )}$ & $\mathbf{( 7 2 1 )}$ & $\mathbf{( 1 5 1 )}$ & $\mathbf{( 3 9 5 )}$ & $\mathbf{( 4 5 5 )}$ & $\mathbf{( 4 6 5 )}$ \\
\hline Chez mes parents & $50,5 \%$ & $54,8 \%$ & $46,2 \%$ & $92,1 \%$ & $77,5 \%$ & $44,6 \%$ & $20,0 \%$ \\
\hline Chez mes beaux-parents & $1,2 \%$ & $1,5 \%$ & $1 \%$ & $7 \%$ & $5 \%$ & $2,2 \%$ & $1,1 \%$ \\
\hline Dans mon propre logement & $42,4 \%$ & $38,1 \%$ & $46,9 \%$ & $3,3 \%$ & $13,7 \%$ & $45,9 \%$ & $76,1 \%$ \\
\hline Dans un appartement partagé avec des amis & $4,6 \%$ & $4,6 \%$ & $4,6 \%$ & $2,6 \%$ & $6,3 \%$ & $6,2 \%$ & $2,2 \%$ \\
\hline Hébergé par d'autres personnes & $9 \%$ & $8 \%$ & $1 \%$ & & $1,3 \%$ & $1,1 \%$ & $6 \%$ \\
\hline Dans une résidence étudiante & $3 \%$ & $3 \%$ & $4 \%$ & $1,3 \%$ & $0,8 \%$ & & \\
\hline Total & $100 \%$ & $100 \%$ & $100 \%$ & $100 \%$ & $100 \%$ & $100 \%$ & $100 \%$ \\
\hline
\end{tabular}

Source: Injuve, 2005, questionnaire auprès des jeunes espagnols.

Tableau 7 : Réponses à la question :

«Pourquoi ne vivez-vous pas où vous souhaitez vivre?» (réponse spontanée)

\begin{tabular}{|l|r|r|r|r|r|r|r|}
\hline & \multicolumn{1}{|c|}{ Total } & \multicolumn{2}{|c|}{ Sexe } & \multicolumn{4}{|c|}{ Tranches d'âge (4) } \\
\hline & & Hommes & Femmes & $18-19$ & $20-24$ & $25-29$ & $30-34$ \\
\hline $\mathbf{N}=$ & $\mathbf{( 6 4 4 )}$ & $\mathbf{( 3 4 9 )}$ & $\mathbf{( 2 9 5 )}$ & $\mathbf{( 8 9 )}$ & $\mathbf{( 2 5 3 )}$ & $\mathbf{( 2 0 7 )}$ & $\mathbf{( 9 5 )}$ \\
\hline Raisons économiques & $58,2 \%$ & $59,6 \%$ & $56,6 \%$ & $59,6 \%$ & $58,1 \%$ & $60,9 \%$ & $51,6 \%$ \\
\hline Prix du logement & $9,9 \%$ & $10,9 \%$ & $8,8 \%$ & $2,2 \%$ & $8,7 \%$ & $13,0 \%$ & $13,7 \%$ \\
\hline Conditions de travail & $9,9 \%$ & $9,5 \%$ & $10,5 \%$ & $11,2 \%$ & $8,3 \%$ & $11,1 \%$ & $10,5 \%$ \\
\hline Suis toujours étudiant & $12,0 \%$ & $10,3 \%$ & $13,9 \%$ & $23,6 \%$ & $19 \%$ & $3,9 \%$ & \\
\hline Le ferai bientôt & $4 \%$ & $4 \%$ & $4,1 \%$ & & $3,2 \%$ & $5,3 \%$ & $7,4 \%$ \\
\hline Réponses autres & $2,8 \%$ & $3,2 \%$ & $2,4 \%$ & $2,2 \%$ & $2 \%$ & $3,4 \%$ & $4,2 \%$ \\
\hline Absence de réponse & $3,1 \%$ & $2,6 \%$ & $3,7 \%$ & $1,1 \%$ & $0,8 \%$ & $2,4 \%$ & $12,6 \%$ \\
\hline Total & $100 \%$ & $100 \%$ & $100 \%$ & $100 \%$ & $100 \%$ & $100 \%$ & $100 \%$ \\
\hline
\end{tabular}

Source: Injuve, 2005.

Ce schéma culturel spécifique mis à part, de nombreux jeunes donnent des justifications économiques au fait de vivre chez leurs parents, la principale étant la difficulté à trouver un emploi. (voir tableau 7):

Le prix des logements ne représente que $10 \%$ des réponses et arrive en troisième position. Cependant, les raisons économiques incluent dans une certaine mesure à la fois les prix du logement et la précarité du travail (ou le manque de travail), obligeant ainsi les jeunes gens à demeurer chez leurs parents. Nous pouvons faire l'hypothèse que près de $80 \%$ des jeunes interrogés considèrent ces deux aspects (prix élevés du logement et emplois précaires) comme les raisons principales de leur non-émancipation et de leur manque d'indépendance (NAVARRETE, 2006).
La transition du fordisme inachevé au postfordisme en Espagne a engendré de profondes transformations sociales, le postfordisme ayant eu pour effet d'élargir le fossé entre de nouvelles classes moyennes, puissantes et devenues riches, et les franges fragiles et moins bien assises de la population, qui luttent pour conserver leurs emplois précaires. La précarité touche un grand nombre de groupes sociaux, en particulier les jeunes; elle n'est pas liée toutefois aux seuls emplois, mais également au logement, à la fondation d'un foyer, etc. Nous pouvons même parler de précarité de la vie en général: il semble n'exister aucun lien qui soit stable, dans cette modernité liquide (BAUMAN, 2000). Il s'agit là d'une précarité au sens le plus vital, du niveau de l'analyse biopolitique.

Les jeunes générations semblent ne pas faire face à cette précarité, bien que directement touchées. L'attitude affichée par beaucoup de ces générations est celle de l'apolitisme (ou du conservatisme, selon le point de vue): elles font face à ces importants problèmes en affichant une sorte de consumérisme amnésique. En lieu et place d'un mouvement fort et organisé visant à lutter contre la situation, la société de consommation espagnole des dernières 
décennies, a propulsé l'Espagne au premier rang pour la consommation de cocaïne et de substances similaires. Le phénomène de beuverie (Botellón) est devenu une part importante de l'identité des jeunes, mais il est intéressant de noter que le fait d'aller faire des «achats », en est également devenu un aspect significatif. Il est de nos jours normal pour les parents appartenant aux classes moyennes et à la bourgeoisie d'acheter à leurs enfants toutes sortes de gadgets technologiques et autres biens de consommation, y compris des voitures; dans le même temps, les classes sociales inférieures éprouvent plus de difficultés à faire face à ce mode culturel de consommation.

Le chômage, chez les jeunes, est considéré comme un problème crucial et ce, pour plusieurs raisons. Cela signifie non seulement un drame personnellement vécu mais également une perte en capital humain. D'autres conséquences se sont également manifestées, comme le processus d'assouplissement du marché du travail visant d'abord les jeunes, puis qui s'est appliqué à tous. Toutes les réformes ciblant le marché du travail ont accru la précarité des jeunes gens, mais cette précarité ne s'est pas cantonnée à eux. Selon le sociologue espagnol Andrés Bilbao, le chômage chez les jeunes a été l'alibi permettant de réduire les droits sociaux et de désagréger les relations du travail (Bilbao, 1989). Ce schéma du marché du travail (s'établissant autour d'une séparation distincte entre les groupes bénéficiant de postes intéressants, de contrats fixes et pouvant investir dans un logement, etc. et ceux grevés d'hypothèques élevées, disposant d'emplois non intéressants et vivant en situation précaire) est en partie masqué par un mode de consommation partagé par presque tous, au sein de la société, (exception faite des catégories les plus pauvres), bien que dans des sens différents. Ce schéma est basé sur l'imitation des modèles de consommation accélérée des groupes sociaux de référence: pour ce faire, ces groupes doivent abandonner toute idée d'épargne et toute stratégie de consommation sur le long terme. Par conséquent, la plupart des groupes sociaux souffrant de précarité (et d'autres moins précaires) semblent être influencés par des normes de consommation qui les poussent à dépenser plus qu'ils ne gagnent réellement, d'où une augmentation des crédits et des dettes. D'autre part, ce fait semble endiguer toute résistance de ces groupes à l'égard des mauvaises conditions de travail, exception faite de certains groupes sociaux très spécifiques.

Cette société postmoderne est toutefois confrontée à un futur problématique; il lui sera difficile de survivre. En dépit de la croissance du PIB, il est clair que ce modèle repose sur la solidarité entre les générations. Les générations qui vivaient à l'époque du fordisme avaient non seulement la possibilité d'avoir accès aux biens de consommation, mais elles pouvaient également devenir propriétaires de leurs propres logements, acheter leurs propres véhicules et épargner, du fait de la redistribution des richesses prévalant sous le modèle keynésien, dit redistribution keynésienne. Néanmoins, la crise liée à ces processus de redistribution a eu une conséquence: de nos jours, les nouvelles générations ont un revenu réel plus faible et leur niveau de vie, en grande partie, (dans le cas de l'Espagne) est dû au transfert de revenus s'opérant entre générations plus anciennes et générations plus jeunes. Les jeunes générations consomment car elles reçoivent de l'argent de leurs parents, mais en même temps, elles dépensent l'argent de leurs futurs enfants, consommant de plus en plus et sollicitant des crédits (argent futur) pour y parvenir. Le défi tient à ce qui va se passer pour les générations à venir, les générations actuelles s'étant tellement endettées (particulièrement du fait des coûts ahurissants des prêts immobiliers) et vivant dans une situation tellement précaire, qu'elles se trouveront dans l'impossibilité d'apporter quelque soutien aux générations futures. Nous pouvons ainsi nous interroger et mettre en doute la durabilité réelle de ce modèle.

Avec le postfordisme, en Espagne, c'est également un pan sombre qui s'est dévoilé: derrière les chiffres macro-économiques, qui placent le pays au rang de huitième économie mondiale, ce sont aussi les emplois précaires ou mal rémunérés, la spéculation ayant rendu impossible l'accès au logement et des habitudes de consommation frénétiques qui viennent compliquer et assombrir les perspectives d'avenir des générations futures. Il est important de réfléchir à ces problèmes et de retrouver des discours et des pratiques de solidarité pouvant faire que le futur de cette société soit différent, et dépasse ces sombres perspectives. 


\section{Bibliographie}

Albiol L., Camps L., Goerlich J.M. (1998), La reforma laboral de 1997. Valencia, Tirant lo Blanch.

Alonso L.E. (1999), Trabajo y ciudadanía: estudios sobre la crisis de la sociedad salarial, Madrid, Trotta.

Alonso L.E. (2001), Trabajo y posmodernidad: el empleo débil, Madrid, Fundamentos.

Alonso L.E. (2007), La crisis de la ciudadanía laboral, Barcelona, Anthropos.

Alonso L.E., Conde F. (1997), Historia del consumo en España, Madrid, Debate ( $2^{\circ}$ ed.).

Alonso L.E., Martinez Lucio M. (Eds.) (2006), Employment Relations in a Changing Society: Assessing the PostFordist Paradigm. Basingstoke, Palgrave Macmillan.

Bauman Z. (2000), Liquid Modernity, Cambridge, Polity.

BAylos A. (coord.) (1996), La Reforma Laboral de 1994, Cuenca, Ediciones de la Universidad de Castilla - La Mancha.

Bilbao A. (1989), "La utilización ideológica de los jóvenes", in Torregrosa, J.R. et al., Juventud, trabajo y desempleo: un análisis psicosociológico, Madrid, Ministerio de Trabajo y Asuntos Sociales, pp. 57-65.

Casson M. (1979), Youth Unemployment, London, MacMillan.

Colectivo IoÉ (2008), Barómetro social de España. Análisis del período 1994-2006, Madrid, Traficantes de Sueños.

Cortès Alcala L. (2005), "La crisis de la vivienda". Documentación Social, 138: pp. 81-100.

Cuadrado J.R. (1990), "La expansión de los servicios en el contexto del cambio estructural de la economía española”, Papeles de Economía Española, 42: pp. 98123.

Espido Freire L. (2006), Mileuristas, Barcelona, Ariel.

EtXeZARreta M.(1991), “La economía política del proceso de acumulación”, in Etxezarreta, M. (coord.), La reestructuración del capitalismo en España 1970-1990, Barcelona, Icaria.

InJuve (2005), Sondeo de opinión Juventud y vivienda: principales conclusiones, Madrid, Injuve.

Koch M.(2006), Roads to Post-Fordism: Labour Market and Social Structures in Europe, Aldershot, Ashgate.

LAPARRA M. (2006), La construcción del empleo precario, Madrid, Fundación FOESSA-Cáritas.

LEAL J. (1987), "El boom inmobiliario. Precios altos para rentas bajas", Alfoz, 46: pp. 23-84.
LeAL J. (2002), "Retraso de la emancipación juvenil y dificultad de acceso de los jóvenes a la vivienda", in Iglesias de Ussel, J. et al. (2002), La sociedad: teoría e investigación empírica. Estudios en homenaje a José Jiménez Blanco, Madrid, CIS.

LEAL J. (2003), "Segregación social y mercado de vivienda en las grandes ciudades", Revista Española de Sociología, 2 : pp. 59-75.

LEAL J. (2004), "El diferente modelo residencial de los países del Sur de Europa: El mercado, las viviendas, la familia y el Estado", Arxius, 10: pp. 11-37.

LeAl J., Cortès L.(1995), La dimensión de la ciudad, Madrid, CIS.

Lopez Calle P., Castillo J.J. (2004), Los hijos de las reformas laborales, Madrid, UGT-Madrid.

Miguelez F., Prieto C. (dir. y coord.) (1999), Las relaciones de empleo en España, Madrid, Siglo XXI.

Munoz Del Bustillo R. (1993), "Salarios desempleo y calidad de empleo", en AA. VV., La larga noche neoliberal, Barcelona, Icaria.

Munoz J., Roldan S., Serranon A. (1978), La internacionalización del capital en España, Madrid, Editorial Cuadernos para el Diálogo.

Navarrete Moreno L. (2006), Jóvenes adultos y consecuencias demográficas 2001-2005, Madrid, Injuve.

Observatorio Joven del Empleo en Espana (2007), Temporalidad en el empleo y mercado de trabajo para los jóvenes en España, Madrid, Consejo de la Juventud.

OCDE (1984), El paro juvenil: causas y consecuencias, Madrid, Ministerio de Trabajo y Seguridad Social.

Perez S., Hidalgo A., Calderon M.J. (2006), La economía de las personas jóvenes, Madrid, Injuve.

SAnton A. (2003), "Jóvenes de larga duración : biografías laborales de los jóvenes españoles en la era de a flexibilidad informacional", Revista Española de Sociología, 3 : pp. 87-98.

Santos A. (2005), "Las políticas de empleo en tiempos de flexibilidad", in Arxius de Ciències Socials, 12/13 : pp. 169-177.

ToHARIA L. (1986), "Un fordismo inacabado entre la transición política y la crisis económica: España", in Boyer, R. (Ed.), La flexibilidad del trabajo en Europa, Madrid, Ministerio de Trabajo y Seguridad Social.

UGT-MADRID (2004), El problema de la subcontratación: precariedad laboral, desregulación y desorden productivo, Tribuna Sindical marzo 2004. Madrid. 